Supplement of Atmos. Meas. Tech., 14, 1715-1732, 2021

https://doi.org/10.5194/amt-14-1715-2021-supplement

(c) Author(s) 2021. This work is distributed under

the Creative Commons Attribution 4.0 License.

(c) (1)

\title{
Atmospheric \\ Measurement \\ Techniques
}

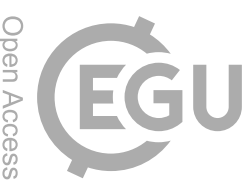

Supplement of

\section{A Compact Rayleigh Autonomous Lidar (CORAL) for the middle atmosphere}

Bernd Kaifler and Natalie Kaifler

Correspondence to: Bernd Kaifler (bernd.kaifler@dlr.de)

The copyright of individual parts of the supplement might differ from the CC BY 4.0 License. 


\section{S1 The effect of thermal expansion of the telescope}

Thermal expansion and contraction of the telescope, most importantly in the $\mathrm{z}$-axis or focus direction, is minimized by using carbon fiber tubes as structural member and spacer between the mirror cell and the telescope spider. The coefficient of thermal expansion of carbon fibers is $-0.1 \times 10^{-6} \mathrm{~K}^{-1}$ along the fiber and $21 \times 10^{-6} \mathrm{mK}^{-1}$ across. The tubes we used have $90 \%$

5 of the fibers aligned along the tube and $10 \%$ across for increased torsion stability. Thus, we assume an average coefficient of $2.09 \times 10^{-6} \mathrm{~m} \mathrm{~K}^{-1}$. Given the focal length of $1.524 \mathrm{~m}$, a change in temperature of $50 \mathrm{~K}$ causes a focus shift of $160 \mu \mathrm{m}$.

We can estimate the impact of the temperature induced focus shift on the coupling efficiency as follows. First, we assume that the facet of the fiber with core diameter $a$ is fully and equally illuminated by the telescope mirror. The half angle of the illuminating light cone is then given by

$10 \tan \beta=\frac{d}{2 f}$.

Next we calculate the fraction the diameter of the illuminated disk increases when the disk, previously at the position of the fiber end, is moved downward by $b=\alpha f$ where $\alpha=2.09 \times 10^{-6} \mathrm{~K}^{-1}$ is the coefficient of thermal expansion:

$\frac{a^{\prime}}{a}=\frac{(2 x+b) \tan \beta}{2 x \tan \beta}=1+\frac{b}{2 x}$.

Looking at Fig. S1, the angle $\beta$ can also be expressed as

$15 \tan \beta=\frac{x}{2 a}$.

Eliminating $\tan \beta$ in this equation using relation (S1) and substituting the result into relation (S2) yields

$\frac{a^{\prime}}{a}=1+\frac{b d}{2 a f}$

Substituting the thermal expansion $b=\alpha f$ into this equation results in

$\frac{a^{\prime}}{a}=1+\frac{\alpha d}{2 a}=1+1.21 \times 10^{-3} \mathrm{~K}^{-1}$.

20 Hence, we expect a $6.0 \%$ increase in the diameter of the illuminated disk for a $50 \mathrm{~K}$ change in temperature. However, the fraction of the light coupled into the fiber core decreases with the diameter squared i.e. a $6.0 \%$ increase in diameter results in a net loss of only $0.36 \%$. In reality, as will be shown in the next section, the signal loss is much smaller because a) the laser beam underfills the FOV of the telescope and b) more energy is concentrated in the center because of the Gaussian beam profile. We conclude that thermal expansion of the telescope has a negligible influence on the performance of the lidar.

25 When setting up the lidar, we scan the vicinity of the expected focus position by slowly moving up and down the fiber end using the focus motor. Because the motor is equipped with encoders, we can record the strength of the detected lidar signal in the altitude range 40-50 km as function of position. Repeated scans are performed and measurements averaged in order to reduce the effect of potential changes in atmospheric transmission during the scans. We find the optimum focus position by fitting the position of the signal maximum.

\section{S2 Range-dependent effects}

In order to evaluate range-dependent effects, we look at the function $P(z)$ which describes the fraction of the collected scattered light with $1 / \mathrm{e}^{2}$ beam radius $w$ passing through an aperture with radius $r$ as function of altitude $z$ :

$P(z)=1-\exp \left(-\frac{2 r^{2}}{w(z)^{2}}\right)$ 
As aperture we define the core of the optical fiber and set $r=0.5 a$. The radius of the beam of scattered laser light at the

$w=\frac{\epsilon}{\mathrm{FOV}} \frac{a^{\prime}}{2}$

where $\epsilon$ is the full-angle divergence of the imaged laser beam taken at $1 / e^{2}$ points and FOV is the full-angle of the telescope FOV. Because the beam divergence of $170 \mu \mathrm{rad}$ was measured at $1 / e$ points we have to scale the value by 1.41 . In addition, we add the effective broadening due to the pointing jitter of the laser $(25 \mu \mathrm{rad})$ and broadening due to the point spread function of the telescope ( $30 \mu \mathrm{rad}$ since the spot size of $60 \mu \mathrm{rad}$ refers to the $2 \sigma$-value) and arrive at the beam radius of $0.408 a^{\prime}$ at the facet of the fiber.

Even if the laser beam is completely within the FOV of the telescope (full geometric overlap is achieved at $4.82 \mathrm{~km}$ altitude), signal may be lost due to defocusing of the telescope at low altitudes. To estimate this range-dependent signal loss, we first calculate the focus shift associated with moving an object (scattered laser light) from infinity to the near vicinity of the telescope and then evaluate how this focus shift affects the coupling efficiency of telescope and fiber in a similar way as was done when analyzing the effect of thermal expansion.

Looking at Fig. S1, the angular change of the object rays is given by

$\gamma=\frac{d}{2 z}$.

with $z$ being the altitude. Here we have used the small-angle approximation $\tan \Theta \approx \Theta$. Moving the object closer i.e. increasing $\gamma$ results in an increase of the focal length of the telescope by $\delta$. The new angle

$\beta^{\prime}=\beta-\gamma$

is defined by relating the new focal length to the diameter of the telescope mirror as given by

$\tan \beta^{\prime}=\frac{d}{2(f+\delta)}$

Solving this equation for $\delta$ and substituting relations (S1), (S8) and (S9) yields

$55 \delta=\frac{d}{2 \tan \left(\tan ^{-1}\left(\frac{d}{2 f}\right)-\frac{d}{2 z}\right)}$.

Now we solve Eqn. (S4) for $a^{\prime}$, replace $b$ with the focus shift $\delta$, and together with Eqn. (S7) substitute the result into Eqn. (S6):

$P(z)=1-\exp \left(-2 \frac{\mathrm{FOV}^{2}}{\epsilon^{2}} \frac{1}{\left(1+\frac{\delta d}{2 a f}\right)^{2}}\right)$

Note that the exponential depends on the inverse of the filling factor $\epsilon \mathrm{FOV}^{-1}$ that describes the ratio of effective laser beam divergence and the telescope FOV, and the scaling term that takes into account the broadening of the imaged laser spot caused by the focus shift. Fig. S2 shows the altitude-dependence of $P$ and also includes two additional gray lines, the upper line marking the altitude $(45 \mathrm{~km})$ for which the focus position position was experimentally determined as described above, and the lower line marking our threshold of $15 \mathrm{~km}$ altitude. The difference in the normalized encircled energy between the two gray lines is $1.35 \%$. In order to assess the impact of this range-dependent effect on retrieved temperature, we multiplied photon count profiles with the correction term $P^{-1}(z)$ before running the retrieval. Fig. S3 shows the differences between retrieval runs with and without this correction term for the data shown in Fig. 8 of the paper. Note that we increased the integration 
time to $60 \mathrm{~min}$ in order to reduce the standard errors. As evident from Fig. S3, defocusing of the telescope results in a low bias of less than $-0.4 \mathrm{~K}$ above $40 \mathrm{~km}$ altitude. The bias increases to $-0.6 \mathrm{~K}$ at $25 \mathrm{~km}$ and reaches $-0.95 \mathrm{~K}$ at $15 \mathrm{~km}$. Below, the temperature bias increases rapidly, as indicated by the steepening curve in Fig. S3. In order to limit this bias to less than $1 \mathrm{~K}$, retrieved profiles are truncated at $15 \mathrm{~km}$ altitude. We note that the actual bias is likely smaller than suggested by Fig. 70 S3. A detailed comparison study which is currently in preparation for publication analyzes CORAL temperature profiles and ECMWF profiles in the lower stratosphere.

So far, besides tests, we do not attempt to correct this range-dependent effect in our implementation of the temperature retrieval because, in addition to the above theoretical considerations, a more thorough experimental study of the overlap function is needed. 


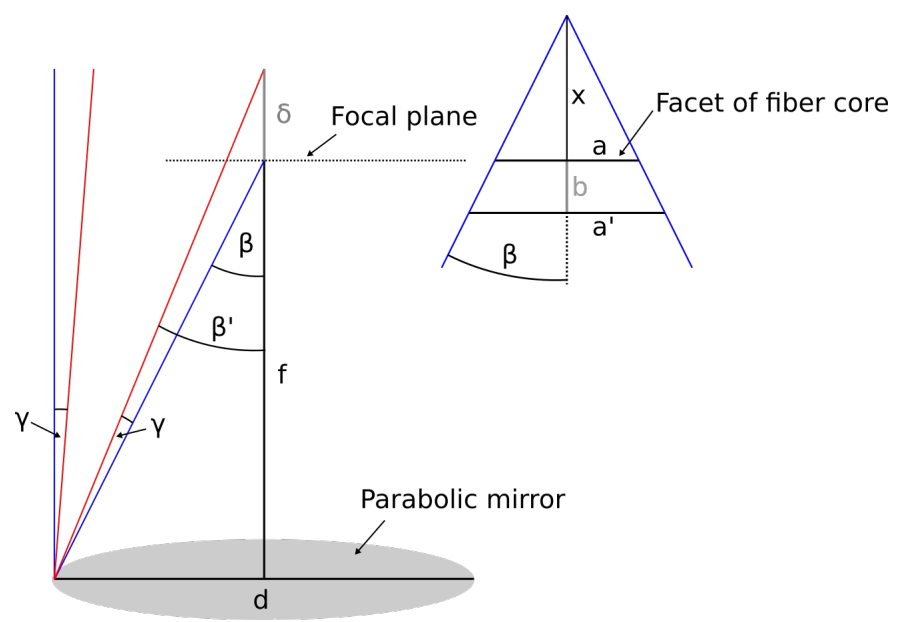

Figure S1. Sketch showing the geometry of rays originating from infinity (blue) and near distance (red) reflected off the telescope mirror.

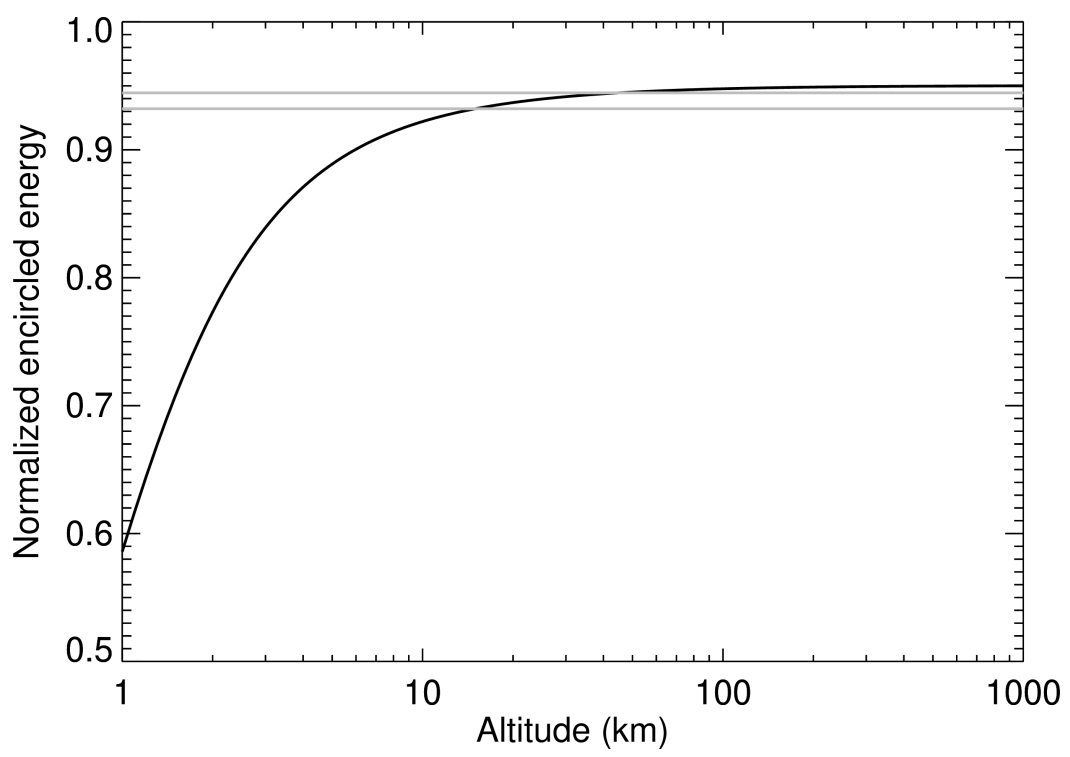

Figure S2. Fraction of the energy passing through the aperture of the fiber core as function of altitude. The gray lines mark points where the curve crosses the focusing altitude $45 \mathrm{~km}$ and the threshold altitude $15 \mathrm{~km}$. 


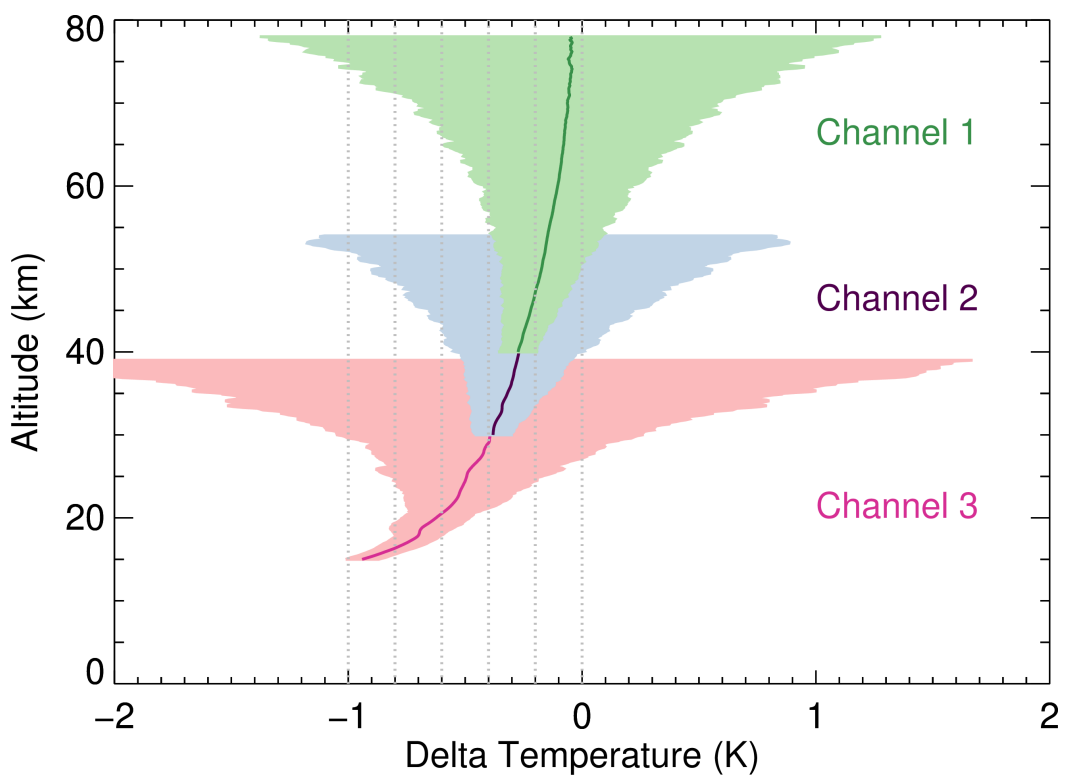

Figure S3. Differences between corrected and uncorrected temperature profiles for the data shown in Fig. 8 of the paper with the integration time increased to $60 \mathrm{~min}$. The shaded areas mark standard errors reported by the retrieval. 